\title{
May 2019 Imaging Case of the Month: Asymptomatic Pulmonary Nodules and Cysts in a 47-Year-Old Woman
}

\author{
Michael B. Gotway, MD \\ Department of Radiology \\ Mayo Clinic Arizona \\ Scottsdale, AZ USA
}

Clinical History: A 47-year-old previously healthy woman presented to her new physician for a routine physical examination. The patient had no complaints. The patient's physical examination showed normal vital signs and clear lungs; the physical examination was essentially unremarkable. The patient's past medical history included a brief smoking history, having quit over 20 years earlier, as well as seasonal allergies. Her past surgical history included an appendectomy nearly 20 years earlier and a hysterectomy for bleeding related to uterine leiomyomas approximately 12 years prior to presentation. The patient was not taking any prescription medications.

Basic laboratory data, including a complete blood count, electrolyte panel, and liver function studies were all within the normal range. An electrocardiogram revealed normal findings. Frontal and lateral chest radiography (Figure 1) was performed.

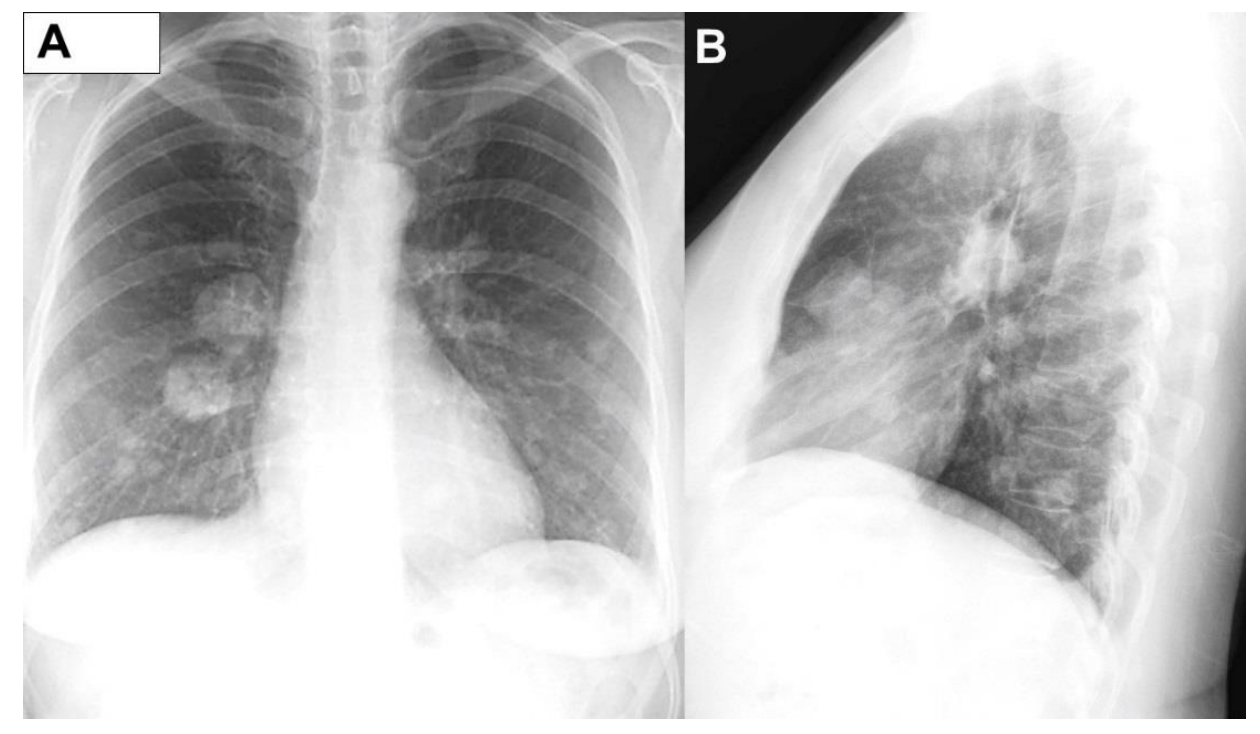

Figure 1. Frontal $(A)$ and lateral $(B)$ chest radiography.

Which of the following statements regarding the chest radiograph is most accurate?

1. The chest radiograph shows mediastinal and hilar lymph node enlargement

2. The chest radiograph shows multifocal nodular pulmonary consolidation

3. The chest radiograph shows multiple, bilateral cavitary nodules

4. The chest radiograph shows multiple, bilateral circumscribed nodules

5. The chest radiograph shows nodular interstitial thickening 


\section{Correct! \\ 4. The chest radiograph shows multiple, bilateral circumscribed nodules}

The chest radiograph shows multiple, bilateral circumscribed nodules, but the nodules do not contain internal lucency- there is no evidence of cavitation. The interstitium does not appear abnormally thickened and lung volumes appear normal. The circumscribed nature of the opacities, and the lack of air bronchograms, indicate that the chest radiographic abnormalities should not be characterized as consolidation. The hilar and mediastinal contours appear normal- no evidence of peribronchial or mediastinal lymphadenopathy is seen.

Which of the following courses of action is the most appropriate next step for the management of this patient?

1. ${ }^{18}$ FDG-PET scanning

2. Bronchoscopy with transbronchial biopsy

3. Comparison to prior thoracic imaging studies

4. Percutaneous transthoracic fine needle aspiration biopsy

5. Serial thoracic CT to assess for growth or change in the size of the nodules 


\section{Correct! \\ 3. Comparison to prior thoracic imaging studies}

As a general rule, whenever chest imaging studies show an abnormality, if comparison images are available, they should be reviewed. When such comparison studies show stable findings, a less aggressive posture regarding the evaluation of the imaging abnormalities is often warranted, and, on occasion, imaging abnormalities may be entirely dismissed as benign when long-term stability is demonstrated. In this patient's case, it is unlikely that the multiple nodules detected at chest imaging will be simply dismissed through comparison to prior studies, but the approach to the current imaging findings may be altered by the results of such comparison studies. Thoracic CT would be a reasonable option to pursue while prior studies are being located, but thoracic CT was not offered as one of the choices for the question. Rather, serial thoracic CT assessment for nodule growth was offered as a choice, but, based on the size and number of the nodules, this management strategy is not the best choice. Tissue sampling procedures are reasonable considerations as well, but premature at this point. Finally, ${ }^{18}$ FDG-PET may prove of use for the evaluation of this patient at some point, but the results of ${ }^{18} \mathrm{FDG}-\mathrm{PET}$ are unlikely to alter the approach to the chest radiographic findings- if the nodules are metabolically active, they will likely be characterized by thoracic CT, and the same will occur if little metabolic activity is seen within these nodules. Furthermore, in the setting of nodule assessment, ${ }^{18}$ FDG-PET scanning is typically reserved for patients in whom the nodules are found to be indeterminate and non-specific following thoracic CT characterization.

A frontal chest radiograph from 3 years earlier (Figure 2) was located for comparison.

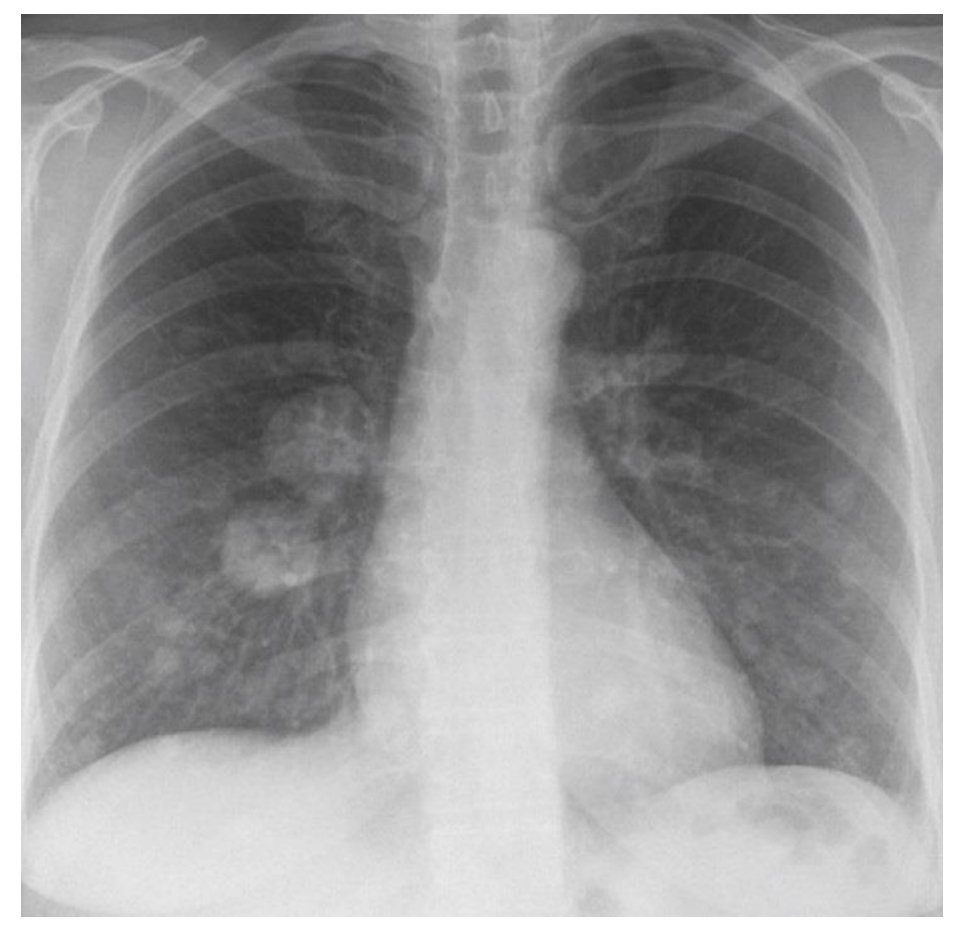

Figure 2. Frontal chest radiograph performed 3 years earlier. 
Which of the following statements regarding the chest radiograph is most accurate?

1. The chest radiograph shows multiple, bilateral circumscribed nodules have increased in size on the current study (Figure 1) compared with the previous examination (Figure 2)

2. The chest radiograph shows that the multiple, bilateral circumscribed nodules are unchanged on the prior examination (Figure 1) compared with the current study (Figure 2)

3. The chest radiograph shows that the multiple, bilateral circumscribed nodules are new on the prior examination (Figure 2) compared with the current study (Figure 1)

4. The chest radiograph shows that the multiple, bilateral circumscribed nodules have decreased in size on the current study (Figure 1) compared with the previous examination (Figure 2)

5. The chest radiograph shows that the multiple, bilateral nodules were previously cavitary 


\section{Correct!}

2. The chest radiograph shows that the multiple, bilateral circumscribed nodules are unchanged on the prior examination (Figure 1) compared with the current study (Figure 2)

The prior frontal chest radiograph from 3 years earlier (Figure 2) shows multiple, bilateral circumscribed nodules have not measurably changed from the presentation chest radiograph (Figure 1). There is no evidence of cavitation within the nodules.

Which of the following courses of action is the most appropriate next step for the management of this patient?
1. ${ }^{18}$ FDG-PET scanning
2. ${ }^{68} \mathrm{Ga}-$ Dotatate scanning
3. Bronchoscopy with transbronchial biopsy
4. Enhanced thoracic MRI
5. Unenhanced thoracic CT 


\section{Correct! \\ 5. Unenhanced thoracic CT}

Unenhanced thoracic CT is the next most appropriate step for the characterization of the pulmonary opacities in this patient. Enhanced thoracic MRI can be useful for cardiovascular evaluation and the evaluation of chest wall and mediastinal masses, but the rather poor signal typical of the pulmonary parenchyma at MRI renders MRI less suitable than CT for pulmonary nodule evaluation. ${ }^{68} \mathrm{Ga}$-Dotatate scanning is typically employed for the evaluation of neuroendocrine malignancies, which may ultimately prove useful in this patient should that diagnosis be established, but is premature at this point. As noted previously, ${ }^{18} \mathrm{FDG}-\mathrm{PET}$ scanning is commonly reserved for patients in whom pulmonary nodules are found to be indeterminate following thoracic CT characterization. Bronchoscopy with transbronchial biopsy is not an unreasonable choice, but this procedure may be best utilized following CT characterization of the nodules. If nothing else, the bronchoscopic procedure may be more appropriately targeted using the information obtained at thoracic CT.

The patient subsequently underwent thoracic CT (Figure 3) for further characterization of the pulmonary nodule seen at chest radiography.

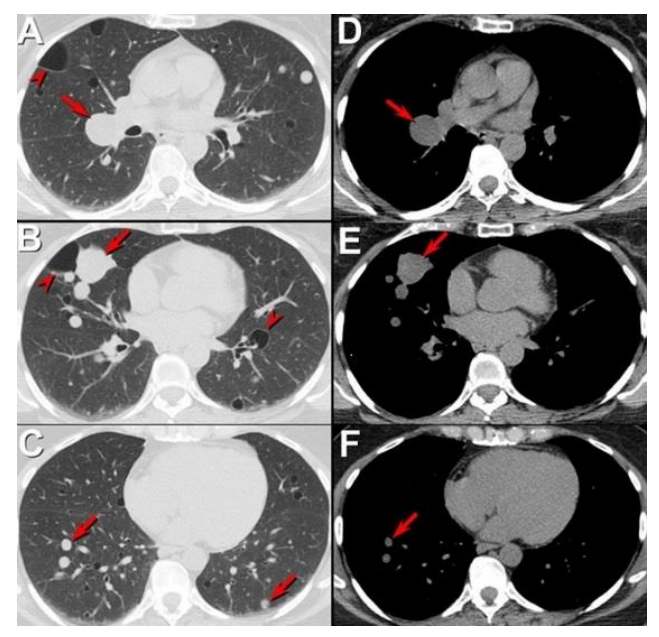

Figure 3. Thoracic CT displayed in lung (A-C) and soft tissue (D-F) windows.

Regarding the thoracic $\mathrm{CT}$, which of the following statements is most accurate?

1. The thoracic CT shows multiple nodules with "feeding vessels" suggestive of arteriovenous malformations

2. The thoracic CT shows multiple nodules with surrounding ground-glass opacity halos

3. The thoracic CT shows multiple, bilateral circumscribed cavitary nodules

4. The thoracic CT shows numerous small nodules consistent with a "miliary" pattern

5. The thoracic CT shows variably-sized, circumscribed, non-calcified nodules and cysts bilaterally 


\section{Correct! \\ 5. The thoracic CT shows variably-sized, circumscribed, non-calcified nodules and cysts bilaterally}

The thoracic CT shows multiple, bilateral non-calcified circumscribed pulmonary nodules as well as scattered thin-walled pulmonary cysts, some of which are closely associated with the nodules. The nodules range in size from a few millimeters to nearly $3 \mathrm{~cm}$ - this size range is inconsistent with miliary disease, as "miliary" nodules usually measure 1-2 $\mathrm{mm}$ in size. None of the nodules exhibit an association with enlarged vessels, and therefore the morphology of these nodules is not suggestive of arteriovenous malformation. The nodules are largely circumscribed and no ground-glass opacity, a finding that often suggests a hemorrhagic lesion, is seen surrounding the nodules (i.e., there is no "ground-glass opacity "halo"). None of the nodules show cavitation, although several cysts are present bilaterally, some closely associated with the nodules.

Regarding the assessment of the thoracic CT findings, which of the following is most accurate?

1. The nodules are indeterminate as regards their etiology

2. The nodules are most consistent with a benign, non-infectious inflammatory process

3. The nodules are most consistent with a benign, post-infectious process

4. The nodules are most consistent with a highly aggressive process, such as malignancy

5. The nodules are most consistent with benign pulmonary neoplasia 


\section{Correct! \\ 1. The nodules are indeterminate as regards their etiology}

The nodules are technically indeterminate, and, while the incidental detection of an aggressive process, such as malignancy (choice 4), does occasionally occur, this possibility is less likely given that little change in the size of the nodules has been noted over a 3-year period. The nodules could be the result of a number of indolent processes, including infections (particularly fungal infections), benign neoplasms, or rare proliferative lesions, but there is simply no way to distinguish among these various possibilities at this point.

A thoracic CT scan from 5 years earlier (Figure 4) was located.

Figure 4. Axial enhanced thoracic CT performed 5 years prior to presentation.

Regarding the thoracic CT, which of the following statements is most accurate?

1. The previous thoracic CT (Figure 4) shows no evidence of either the nodules or cysts found on the presentation thoracic CT (Figure 3)

2. The previous thoracic CT (Figure 4) shows that the nodules and cysts seen at the presentation thoracic CT (Figure 3) are present and unchanged from 5 years earlier

3. The previous thoracic CT (Figure 4) shows that the nodules and cysts seen at the presentation thoracic CT (Figure 3) have increased in size and number

4. The previous thoracic CT (Figure 4) shows that the nodules and cysts seen at the presentation thoracic CT (Figure 3) have decreased in size and number

5. The previous thoracic CT (Figure 4) shows that the nodules seen at the presentation thoracic CT (Figure 3 ) were all previously cavitary 


\section{Correct!}

3. The previous thoracic CT (Figure 4) shows that the nodules and cysts seen at the presentation thoracic CT (Figure 3) have increased in size and number

The previous thoracic CT from 5 years earlier (Figure 4) again shows multiple circumscribed nodules and thin-walled cysts, but these lesions are considerably fewer in number and smaller in size than at the presentation thoracic CT (Figure 3). One the of the larger cysts, in the right middle lobe, seen at the presentation thoracic CT (Figure 3) can be visualized on the prior CT (Figure 4), considerably smaller on that prior study than at presentation.

At this point, which of the following are appropriate differential diagnostic considerations for this patient?

1. Amyloidosis

2. Lymphangioleiomyomatosis-tuberous sclerosis complex

3. Lymphocytic interstitial pneumonia

4. Papillomatosis

5. All of the above 


\section{Correct! \\ 5. All of the above}

All of the entities listed above could produce nodules with associated cysts and progress in a relatively indolent fashion.

What is the appropriate next step for the evaluation / management of this patient?

1. ${ }^{18}$ FDG-PET scanning

2. Bronchoscopy with transbronchial biopsy

3. Percutaneous transthoracic fine needle aspiration and core biopsy

4. Serial thoracic CT to assess for growth or change in the size of the nodules

5. Surgical lung biopsy 


\section{Correct!}

\section{Percutaneous transthoracic fine needle aspiration and core biopsy}

While the other tissue sampling procedures listed- bronchoscopy with transbronchial biopsy and surgical lung biopsy- may be capable of establishing a diagnosis, percutaneous transthoracic fine needle aspiration and core biopsy may be the best choice among those listed because it is the least invasive method for obtaining a fairly large tissue sample. Bronchoscopy with transbronchial biopsy is less invasive than percutaneous transthoracic fine needle aspiration and core biopsy, but the tissue sample is smaller with the former compared with the latter, and the addition of core biopsy in this circumstance is important, as the diagnosis may not be a cytologicallyevident carcinoma, in which case the histopathological sample provided by core biopsy may prove definitive. Surgical lung biopsy certainly is capable of obtaining the tissue needed for diagnosis, but is more invasive compared with percutaneous transthoracic fine needle aspiration and core biopsy and is potentially not needed to establish a definitive diagnosis. ${ }^{18}$ FDG-PET scanning may prove useful for evaluation of the nodules in this patient, but the lack of tracer utilization within the nodules would not preclude the need to establish a definitive diagnosis, and elevated tracer utilization within the nodules would add no new management-altering information. It could be reasoned that, if some of the nodules showed tracer accumulation at ${ }^{18 F}$ FDG-PET and others did not, tissue sampling should be directed towards the metabolically active lesions, but this approach still leaves tissue sampling as the primary consideration for the next step in management.

Percutaneous fine needle aspiration biopsy and core biopsy of the dominant right middle lobe nodule was performed (Figure 5).

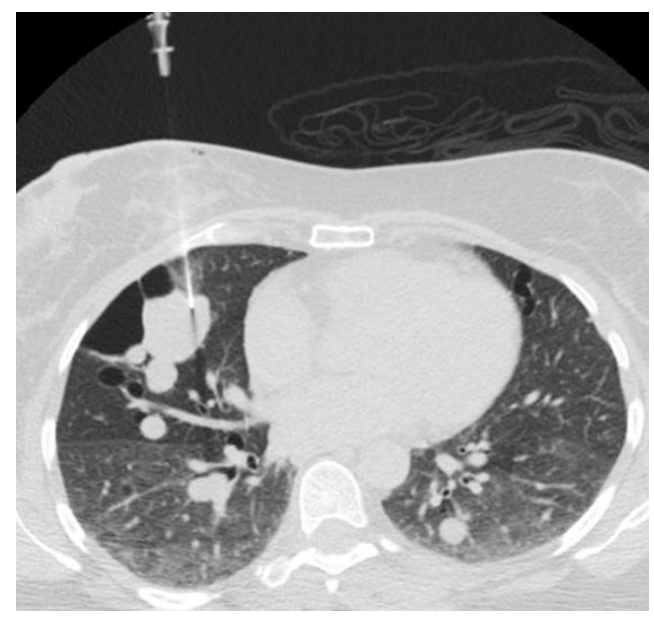

Figure 5. Axial unenhanced thoracic CT shows percutaneous placement of the biopsy needle in the dominant right middle lobe nodule.

The biopsy showed fragments of pulmonary parenchyma entrapped within foci of proliferating fusiform cells containing eosinophilic cytoplasm and oval nuclei. A proliferation of cytologically benign-appearing smooth muscle cells without anaplasia 
was noted, and necrosis, mitotic activity, and pleomorphism were not seen. Immunohistochemical staining showed strong, diffuse nuclear staining for estrogen and progesterone receptors and diffuse cytoplasmic staining for smooth muscle-specific actin, but without staining for CD10 (a B-lymphocyte marker), and only weak activity for vimentin.

In light of these histopathological findings, among the following choices, which piece of historical information is most useful for establishing the diagnosis for this patient?

1. The patient had a basal cell malignancy of the skin resected 4 years earlier

2. The patient had a prior history of hysterectomy

3. The patient has a positive anti-nuclear antibody titer and antineutrophil cytoplasmic antibodies are present

4. The patient has serum protein electrophoresis immunofixation positive for $\lg A$ lambda

5. The patient has thalassemia 


\section{Correct! \\ 2. The patient had a prior history of hysterectomy}

The history of thalassemia would raise the possibility of extramedullary hematopoiesis, but this process most commonly presents as paravertebral, posterior mediastinal nodules or masses and is rarely found within the lung parenchyma itself, and, when in the lung parenchyma, rarely presents as multiple nodules. The pulmonary parenchymal cysts would not be accounted for by extramedullary hematopoiesis also. The nodules and cysts could be seen with pulmonary manifestations of a connective tissue disorder, such as lymphocytic interstitial pneumonia, and the nodules are typical of an antineutrophil cytoplasmic antibody (ANCA)-associated vasculitis. However, as regards the latter, nodules occurring ANCA-associated vasculitis typically show actual cavitation at some point, rather than merely associated cystic change, and the histopathological findings from the biopsy of the right middle lobe lesion are not consistent with a pulmonary manifestation of either a connective tissue disorder or vasculitis. The histopathological findings are consistent with a smooth muscle tumor, but not with a basal cell skin malignancy, which is commonly an indolent skin tumor and would not typically produce pulmonary nodules and cysts. Serum protein electrophoresis immunofixation being positive for IgA lambda would suggest the possibility of light chain deposition disease or amyloidosis, but the histopathological description of the right middle lobe nodule biopsy material is not consistent with this consideration. Combining the indolent behavior of the lung nodules with the histopathological data suggesting a smooth muscle tumor makes the history of prior hysterectomy highly relevant.

Diagnosis: Benign metastasizing leiomyomas presenting as pulmonary nodules and cysts

\section{References}

1. di Scioscio V, Feraco P, Miglio L, Toni F, Malvi D, Pacilli AM, Fasano L, Fabbri M, Zompatori M. Benign metastasizing leiomyoma of the lung: PET findings. J Thorac Imaging. 2009;24(1):41-4. [CrossRef] [PubMed]

2. Ogawa M, Hara M, Ozawa Y, Moriyama S, Yano M, Shimizu S, Shibamoto Y. Benign metastasizing leiomyoma of the lung with malignant transformation mimicking mediastinal tumor. Clin Imaging. 2011;35(5):401-4. [CrossRef] [PubMed]

3. Miller J, Shoni M, Siegert C, Lebenthal A, Godleski J, McNamee C. Benign metastasizing leiomyomas to the lungs: an institutional case series and a review of the recent literature. Ann Thorac Surg. 2016;101(1):253-8. [CrossRef] [PubMed]

4. Abramson S, Gilkeson RC, Goldstein JD, Woodard PK, Eisenberg R, Abramson N. Benign metastasizing leiomyoma: clinical, imaging, and pathologic correlation. AJR Am J Roentgenol. 2001;176:1409-13. [CrossRef] [PubMed]

5. Maredia R, Snyder BJ, Harvey LA, Schwartz AM. Benign metastasizing leiomyoma in the lung. Radiographics. 1998;18:779-82. [CrossRef] [PubMed]

6. Choe YH, Jeon SY, Lee YC, Chung MJ, Park SY, Lee YC, Kim SR. Benign metastasizing leiomyoma presenting as multiple cystic pulmonary nodules: a case report. BMC Womens Health. 2017;17(1):81-5. [CrossRef] [PubMed] 
7. Okabe R, Shoji T, Huang CL. Benign metastasizing leiomyoma of the lung with spontaneous pneumothorax. Thorac Cardiovasc Surg Rep. 2013;2(1):26-8. [CrossRef] [PubMed]

8. Jolissaint JS, Kilbourne SK, LaFortune K, Patel M, Lau CL. Benign metastasizing leiomyomatosis (BML): A rare cause of cavitary and cystic pulmonary nodules. Respir Med Case Rep. 2015;16:122-4. [CrossRef]

9. Aboualfa K, Calandriello L, Dusmet M, Ladas G, Hansell DM, Nicholson AG. Benign metastasizing leiomyoma presenting as cystic lung disease: a diagnostic pitfall. Histopathology. 2011;59(4):796-9. [CrossRef] [PubMed] 